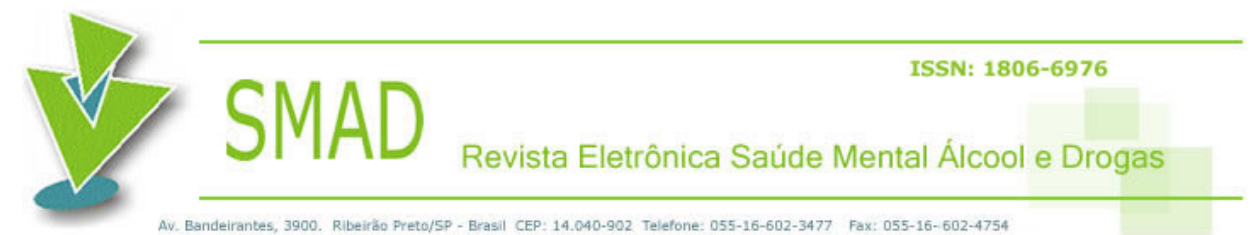

\title{
O ACOMPANHAMENTO TERAPÊUTICO COMO ESTRATÉGIA DE CONTINÊNCIA DO SOFRIMENTO PSÍQUICO
}

Resumo

\section{Ana Celeste de Araújo Pitiá ${ }^{1}$; Manoel Antônio dos Santos ${ }^{2}$}

Este estudo consiste em uma pesquisa bibliográfica que abrange a produção científica na área do Acompanhamento Terapêutico (AT). O objetivo foi avaliar sistematicamente a produção científica indexada nas bases de dados MedLine, PsycINFO e LILACS no período de 1977 a 2001, incluindo também livros e teses produzidos nesse período. Para tanto foram utilizadas as palavras-chave: therapeutic companion e acompanhante terapêutico. Foi feita uma seleção dos resumos que preencheram os critérios de inclusão, cujas publicações foram recuperadas na íntegra. Os resultados indicam que a maioria dos estudos abordou aspectos técnicos do trabalho na clínica do AT, apontando para o perfil desejável do profissional acompanhante terapêutico (at).

Palavras-chave: acompanhamento terapêutico, saúde mental, reabilitação psicossocial.

\section{EL ACOMPAÑAMIENTO TERAPÉUTICO COMO ESTRATEGIA DE CONTINENCIA DEL SUFRIMIENTO PSÍQUICO}

Resumen

Este trabajo consiste en una investigación bibliográfica que abarca la producción científica en el área del Acompañamiento Terapéutico (AT). La finalidad fue evaluar sistemáticamente la producción científica indexada en las bases de datos MedLine, PsycINFO y LILACS, en el período del 1977 al 2001, incluyendo también libros y tesis existentes en aquel período. Para eso, se utilizaron las palabras clave: therapeutic companion y acompañante terapéutico. Se efectuó una selección de los resúmenes que atendieron a los criterios de inclusión, cuyas publicaciones fueron recuperadas por completo. Los resultados indican que la mayoría de los estudios trató de aspectos técnicos del trabajo en la clínica AT, indicando el perfil deseable del profesional acompañante terapéutico (at).

Palabras clave: acompañamiento terapéutico, salud mental, rehabilitación psicosocial.

\section{THERAPEUTIC ACCOMPANIMENT AS A STRATEGY TO CONTAIN MENTAL SUFFERING} Abstract

This paper consists in a bibliographic review of scientific production in the area of Therapeutic Accompaniment (TA). The aim was to systematically assess the scientific production indexed in MedLine, PsycINFO and LILACS between 1977 and 2001, as well as books and theses existing at that time. Therefore, we used the keywords: therapeutic companion and acompañante terapéutico. We selected abstracts that attended to the inclusion criteria, whose publications were recovered in their full version. The results indicate that most articles addressed technical aspects of work at the TA clinic, indicated the desired profile for therapeutic accompaniment (ta) professionals. Key words: therapeutic accompaniment, mental health, psychosocial rehabilitation.

\footnotetext{
${ }^{1}$ Enfermeira, Doutora em Saúde Mental pela EERP/USP, Ribeirão Preto, Professora Pesquisadora junto ao DEPCH da EERP/USP, Ribeirão Preto - PRODOC/CAPES. Psicoterapeuta Corporal Neo-reichiana e Analista Bioenergética pelo Instituto Neo-reichiano Lumen, de Ribeirão Preto. Terapeuta de Acompanhamento Terapêutico. E-mail: aceleste@eerp.usp.br

${ }^{2}$ Psicólogo, Doutor em Psicologia Clínica pelo Instituto de Psicologia da Universidade de São Paulo. Professor doutor do do Departamento de Psicologia e Educação da Faculdade de Filosofia, Ciências e Letras de Ribeirão Preto - FFCLRP-USP. Psicoterapeuta de Casal e Família pelo Instituto Familae-Ribeirão Preto. Bolsista de Produtividade Científica do CNPq. Email: masantos@ffclrp.usp.br
}

SMAD $2006 \quad \begin{aligned} & \text { Volume } \\ & \text { Volumen }\end{aligned} 2 \begin{aligned} & \text { Número } \\ & \text { Numero } 2\end{aligned} \begin{aligned} & \begin{array}{l}\text { Artigo } \\ \text { Artículo } \\ \text { Article }\end{array} \\ & \text { Number }\end{aligned} \quad$ http://www2.eerp.usp.br/resmad/artigos.asp




\section{INTRODUÇÃO}

O primeiro livro sobre Acompanhamento Terapêutico (AT) foi publicado em 1987. Susana Kuras de Mauer e Silvia Resnizky escreveram Acompanhamento Terapêutico e Pacientes Psicóticos: Manual introdutório a uma estratégia clínica, no qual referem o AT a uma prática clínica voltada para a clientela de pacientes psicóticos com esquizofrenia, depressão e risco suicida, psicopatia e drogadição. Nesse livro obtivemos a primeira sistematização dos aspectos técnicos do trabalho do acompanhante terapêutico (at). São enumerados critérios para o profissional que se propõe exercer essa prática: a formação específica e as habilidades individuais de despojamento. As autoras trabalhavam com a terapia de abordagem múltipla - um enfoque multidisciplinar no qual os vários profissionais de saúde estão envolvidos no atendimento. Nessa abordagem o at se inclui na equipe como o agente terapêutico que, nos atendimentos externos à instituição, busca restituir a possibilidade de diálogo entre o lado irracional do indivíduo e o senso comum da sociedade.

O objetivo terapêutico no AT é descrito como significar aquilo que o sujeito expressa com seu adoecer, para que interaja de forma mais saudável em termos biopsicossociais. Os diagnósticos devem ser situacionais, permitindo avaliar a interação do paciente em diferentes contextos: no seu grupo familiar e de amigos, no local de trabalho, na comunidade religiosa, entre outros. A partir disso, pode ser caracterizado o nível de retraimento social a que sua sintomatologia o conduz ${ }^{(1)}$.

A Equipe de Acompanhantes Terapêuticos do Hospital-Dia A Casa publicou dois livros sobre o assunto, resultados de dois Encontros de Acompanhantes Terapêuticos realizados em São Paulo e promovidos por essa mesma equipe. São eles: A rua como espaço clínico: acompanhamento terapêutico e Crise e cidade: acompanhamento terapêutico, ambos editados e publicados em 1991 e 1997, respectivamente. 
O livro A rua como espaço clínico: acompanhamento terapêutico inicia-se com um panorama histórico das concepções de loucura através dos tempos. Os autores percorrem uma trajetória que vai desde a Grécia Antiga, quando a loucura era vista como saber divino, até o século XIX, no qual o enfoque dominante passa a ser psicológico e se concebe o transtorno mental como resultado do ser humano em conflito consigo próprio, gerando sofrimento psíquico. A figura do médico psiquiatra aparece com função ambígüa: eleito para tratar o doente e proteger a sociedade, tenta curar utilizando-se da punição da clausura como estratégia de tratamento ${ }^{(2)}$.

Os autores analisam o século XX e destacam a ineficácia terapêutica de asilos psiquiátricos. Nesse sentido, apresentam as influências históricas sobre os modelos de atendimento em Saúde Mental: o movimento antipsiquiátrico nas décadas de 1950/1960 rompendo a sinonímia cuidado-exclusão; as comunidades terapêuticas, na Inglaterra, Alemanha e EUA; a psiquiatria de setor e a análise institucional na França, criando espaços de acolhimento; a psiquiatria democrática na Itália, inspirada nas idéias de Franco Basaglia que, apontando com radicalidade o enlouquecimento como um produto social, abriu os hospitais psiquiátricos e contribuiu para a desconstrução dos valores da cultura manicomial. No Brasil, na década de 60, essas idéias tomaram corpo com a formação das comunidades terapêuticas no Rio de Janeiro, São Paulo e Porto Alegre, estabelecendo-se então o início da prática do AT como recurso terapêutico.

No processo de teorização sobre a prática clínica no AT os autores definem o trabalho do terapeuta at como forma de acolhimento que opera na produção da (re)colocação do sujeito na realidade urbana, encontrando espaços onde a cidade incorpora a conexão da pessoa à sua organização psíquica e à dinâmica social. As saídas pela cidade, preferencialmente fora dos lugares conhecidos do paciente e já cristalizados pelo hábito, aumentam suas possibilidades de concretizar articulações no social, como sujeitos que exercitam sua potencialidade vital ${ }^{(2)}$.

\begin{tabular}{llll}
\hline SMAD 2006 & $\begin{array}{l}\text { Volume } \\
\text { Volumen } 2\end{array}$ & $\begin{array}{l}\text { Número } \\
\text { Numero } \\
\text { Number }\end{array}$ & $\begin{array}{l}\text { Artigo } \\
\text { Artículo } 07 \\
\text { Article }\end{array}$
\end{tabular} http://www2.eerp.usp.br/resmad/artigos.asp


No segundo livro - Crise e cidade: acompanhamento terapêutico - os autores caracterizam a prática do AT como uma clínica que não consegue operar uma distinção precisa entre acompanhante/acompanhado pelo caráter nômade que a reveste, por ser realizada nas ruas. A relação terapêutica é alvo de constantes elucubrações que marcam a clínica do AT, de tal maneira que uma única abordagem não dá conta de sua complexidade ${ }^{(3)}$. Os autores tentam teorizar aquilo que está acontecendo no empírico da situação terapêutica do acompanhante/acompanhado lado a lado nas ruas. Chamam o AT de clínica do espaço, que pode ser influenciada pelos ecos de Freud, Lacan, Deleuze, Winnicott, Guatari, Reich e tantos outros, estimulando a produção de um desassossego que é próprio às situações de criação.

A dissertação de mestrado de Reis Neto, defendida em 1995, aprofunda o tema do AT, conceituando-o como um campo de intervenção que, antes de ser determinado como uma técnica, pode ser visto por diversos olhares e a partir de diferentes concepções teóricas e ideológicas. É um trabalho que levanta dados históricos sobre a prática do AT no Rio de Janeiro, que se inicia a partir da figura dos auxiliares psiquiátricos - denominação dada desde o Rio Grande do Sul - para chegar nos atuais acompanhantes terapêuticos.

Por meio de um mapeamento histórico o autor traça uma trajetória do trabalho em AT, desde a abordagem múltipla desenvolvida no CETAMP (Centro de Estudio y Tratamiento de Abordaje Múltiple em Psiquiatría, na Argentina) até a Clínica de Vila Pinheiros, no Rio de Janeiro, recorrendo a depoimentos orais dos agentes que estiveram ou estão envolvidos nesse movimento, além de textos e documentos não publicados ${ }^{(4)}$.

Vale ressaltar que os auxiliares psiquiátricos eram leigos ou estudantes da área da saúde preparados dentro das clínicas Vila Pinheiros/RJ e Pinel/RS para acompanhar pacientes em saídas externas. Eles continuaram sendo solicitados por psiquiatras, fora da clínica, mesmo após seu fechamento em 1976, quando o AT passa a ser desenvolvido em um novo contexto o de uma atividade autônoma. O autor não registra progressos científicos na área, contudo

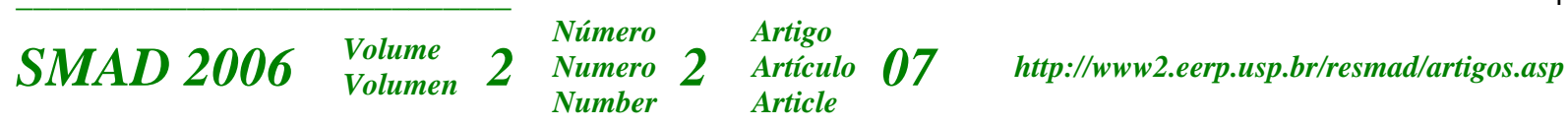


refere que a evolução dos atendimento aos pacientes é decorrente do estabelecimento de um vínculo afetivo e de uma escuta diferenciada, que favorecem uma aproximação à realidade da doença mental. Os acompanhantes que pautavam suas ações no saber psicanalítico canalizaram suas vidas profissionais para a prática em consultório ${ }^{(4)}$.

Na dissertação de mestrado de Débora Sereno, defendida em 1996, a prática do AT é pensada no contexto do tratamento das psicoses, articulando-a a conceitos lacanianos. A autora aponta para dificuldades do ponto de vista de formulações teóricas sobre o tema e afirma que o saber sobre a psicose e uma possível teoria sobre o AT é uma construção, no sentido de montagem. Para ela, esse campo é atravessado por vários saberes sobre a psicose e sobre a própria clínica, que procura articular o mundo do louco à vida cotidiana ${ }^{(5)}$.

Já na dissertação de mestrado de Kleber Barreto, que veio à lume em 1997, a prática do AT é analisada pelo referencial psicanalítico winnicottiano, oferecendo-se uma contribuição ao manejo e enquadre clínicos. O autor se utiliza de exemplos de diversos acompanhamentos realizados, ilustrando-os com aportes teóricos e estabelecendo uma analogia com a estória de Dom Quixote de la Mancha e de seu fiel escudeiro Sancho Pança. Por meio da fundamentação teórica no referencial winnicottiano, o autor enumera conceitos tais como: holding, continência, handling, “apresentação do objeto”, “discriminação e interlocução especular” e “modelo de identificação”(6). Este estudo foi publicado em livro em 1999.

Restritos então a esse limitado número de produções bibliográficas e buscando a construção de um referencial teórico sobre a clínica AT, realizamos uma pesquisa bibliográfica, cujos resultados serão delineados a seguir, tendo em vista também que, naquela ocasião, construíamos um projeto de pesquisa de doutorado sobre o tema.

\section{OBJETIVO}


Avaliar sistematicamente a produção científica constituída por relatos de pesquisa indexados nas bases de dados MedLine, PsycINFO e LILACS no período de 1977 a 2001, considerando-se a escassez de estudos e artigos sobre o tema do AT.

\section{MÉTODO}

Estabelecido o período de 1977 a 2001, foi realizada uma busca sistemática aos estudos capturados por meio das palavras-chaves: therapeutic companion e acompañante terapéutico.

A pesquisa foi feita pela Internet, sendo consultados os sites: www.bireme.br e www.usp.br/sibi. O critério de inclusão adotado previa que os artigos deveriam abordar o Acompanhamento Terapêutico como uma prática clínica que se dá nos espaços urbanos e/ou residenciais, do trabalho ou da escola, podendo estar associada ou não ao trabalho psicoterápico, sendo uma modalidade assistencial oferecida a pessoas em dificuldades psicossociais, ou como parte de um tratamento orientado por uma equipe multiprofissional. Selecionados os resumos que preenchiam esse critério, os artigos levantados foram recuperados na íntegra.

\section{RESULTADOS}

Na base de dados LILACS foram encontrados quatro artigos:

a) Artigo original, também encontrado na base PsycINFO, intitulado "Nuevo modo de investigar en psiquiatria: el acompañamiento terapéutico”, de Beatriz Dorfman Lerner, publicado na Argentina pela Acta de Psiquiatria y Psicologia da América Latina, no ano de 1984. Nele a autora descreve o perfil do at como aquele que, sem ser necessariamente psicólogo, recebe uma instrução adequada para cooperar em uma equipe terapêutica, tendo a função de conter a ansiedade do indivíduo adoecido e dar respostas apropriadas às suas condutas sintomáticas.

$\begin{array}{llll}\text { SMAD } 2006 & \begin{array}{l}\text { Volume } \\ \text { Volumen }\end{array} & \begin{array}{l}\text { Número } \\ \text { Numero } \\ \text { Number }\end{array} & \begin{array}{l}\text { Artigo } \\ \text { Artículo } 07 \\ \text { Article }\end{array}\end{array}$ http://www2.eerp.usp.br/resmad/artigos.asp


A autora revela a importância da formação em AT, descrevendo que o terapeuta at necessita de uma instrução especial sobre a psicopatologia de seus pacientes e precisa dispor de um ego forte para tolerar a ansiedade, a frustração e o assédio à sua integridade física, ao qual se expõe ao entrar em contato com pessoas ansiosas, que se ressentem da falta de discriminação e de adequado controle sobre seus impulsos. Também é necessário à pessoa do at uma alta capacidade de empatia, tato e intuição ${ }^{(7)}$.

b) Artigo original, também de autoria de Beatriz Dorfman Lerner, intitulado: “Como acompañar a un suicida”, publicado na revista Acta Psiquiátrica y Psicológica de América Latina, no ano de 1986. A autora oferece uma orientação para o desempenho do terapeuta at com pacientes que tenham histórico de tentativa de suicídio. Referenciada pelos autores Tabachnik e Farberow, ela apresenta uma espécie de manual para se acompanhar um potencial suicida, mencionando diferentes formas de apresentação das atitudes suicidas: a) o suicídio consumado; b) a intenção do suicídio; c) as idéias suicidas conscientes; d) as idéias suicidas inconscientes expressas por meio de suicídios encobertos (drogas, acidentes); e e) as fantasias suicidas mudas e não visíveis. Destaca a importância do AT como terapêutica preventiva, especialmente para psicóticos que apresentam potencial suicida ${ }^{(8)}$.

Descreve quatro fases para o acompanhamento: Fase 1) a presença do olhar, que possibilita calma, esperança e alívio de intentos auto-destrutivos, que ajudam na superação dos momentos críticos inicias. Fase 2) a escuta, ou seja, a capacidade do acompanhante de conter o sofrimento psíquico do paciente para digeri-lo e devolvê-lo, posteriormente, de maneira elaborada. É importante manter uma postura corporal e gestual relaxada, sem distrações, ao lado de uma disponibilidade expectante, sem significar ansiedade ou exigência, possibilitando assim uma reconstituição histórica do indivíduo. Fase 3) a atitude compreensiva, que possibilita ao acompanhado uma espécie de consentimento para a expressão dos sentimentos que dão ao suicida razões para sofrer a ponto de desejar tirar a

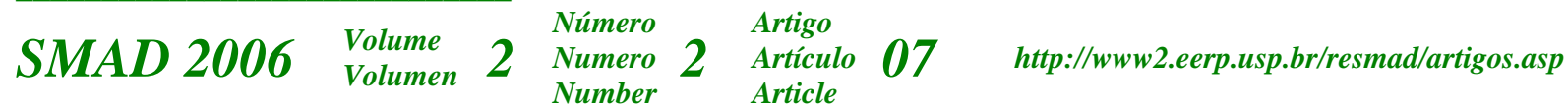


própria vida. Fase 4) o diálogo, um momento de comunicação bidirecional estabelecido entre at-paciente, no qual as indicações terapêuticas para o acompanhamento devem se centrar em uma revisão do projeto de vida do paciente, para o qual é necessário estabelecer uma reestruturação de si próprio ${ }^{(8)}$.

c) Capítulo de livro: “Terapias ressocializantes: o acompanhante terapêutico”, da autoria de Roberto Antonucci, escrito em 1994. Nessa publicação o autor trabalha o conceito da ressocialização através de uma reflexão sobre a psicose na criança e no adulto, destacando os aspectos da personalidade que devem ser reconstituídos ou recuperados dentro da desorganização psíquica em que se encontra o indivíduo. Há uma ênfase atribuída ao efeito do AT realizado com crianças psicóticas, tendo em vista o processo socializador, organizador e estruturante da personalidade.

O autor relata um dado histórico sobre o AT quando faz referência ao atendimento descrito por Sechehaye em 1984, sobre o caso da psicanalista suíça que necessitou treinar uma enfermeira para atender uma de suas pacientes em casa, em 1937. Era uma mulher esquizofrênica, excluída socialmente e que, com o trabalho da psicanalista e da enfermeira (at), após vários anos de tratamento voltou a ter uma vida normal ${ }^{(9)}$.

O autor também referenda a importância do conhecimento teórico no trabalho em AT, quando fala da necessidade do profissional at conhecer as funções do ego para colocação de limites na organização de atividades com o cliente, o que auxiliará na restrição das pulsões instintivas presentes em distúrbios psicóticos. Com o AT espera-se que o psicótico comece (ou volte) a adquirir os contornos de sua personalidade com a experiência de integração. $\mathrm{O}$ at deve ter a capacidade de poder conter o sofrimento psíquico dentro de si e devolvê-lo ao paciente como algo mais elaborado do ponto de vista psíquico e, portanto, mais suportável ${ }^{(9)}$, ao lado da presença de um ego forte para tolerância das angústias. O autor destaca a importância técnica do trabalho em AT por meio do manejo do vínculo terapêutico \begin{tabular}{llll}
\hline SMAD 2006 & $\begin{array}{l}\text { Volume } \\
\text { Volumen }\end{array}$ & $\begin{array}{l}\text { Número } \\
\text { Numero } \\
\text { Number }\end{array}$ & $\begin{array}{l}\text { Artigo } \\
\text { Artículo } 07 \\
\text { Article }\end{array}$
\end{tabular} http://www2.eerp.usp.br/resmad/artigos.asp 
estabelecido com a parte mais sadia ou mais evoluída da personalidade do paciente. Encerra indicando a necessidade de o at estar em psicoterapia individual, bem como ter seu trabalhado avaliado e acompanhado sistematicamente por supervisores qualificados.

d) Artigo da autoria de Paula Ayub: “Do amigo qualificado ao acompanhante terapêutico”, publicado em 1996 no periódico Infanto: Revista de Neuropsiquiatria da Infância e Adolescência. A autora apresenta a origem do trabalho do AT e as transformações do papel do at, através dos anos, definindo alguns critérios de atuação profissional. Demarca a origem do primeiro modelo em AT nas idéias da Antipsiquiatria, nas décadas de 50 e 60, com a quebra dos dogmas de exclusão, isolamento e dor. Faz referência ao estudo de Roberto Antonucci, que pressupõe o início da prática do AT quando uma enfermeira suíça é treinada por uma psicanalista para atender em casa uma de suas pacientes do consultório, em $1937^{(9)}$. Destaca o AT e o contato com a família, considerando que, ao se atender o paciente dentro das residências, parece haver diluição da gravidade do transtorno mental entre os membros da família $^{(10)}$.

A autora refere-se à estruturação da prática em AT como uma nova ocupação surgida nos anos 80, na qual são estabelecidas normas de honorários, perspectivas de tempo/espaço e habilidades pessoais para estabelecimento de um bom vínculo terapêutico. Destaca a amplitude da prática, que não se restringe apenas a indivíduos psicóticos, podendo ser aplicada a pessoas portadoras de deficiências, síndromes e distúrbios do desenvolvimento, sendo realizada na residência ou na rua, levando-se em conta o motivo de pedido do acompanhamento, o diagnóstico do paciente e sua situação dentro do quadro familiar ${ }^{(10)}$.

Na base de dados MedLine foram encontrados 10 estudos, sendo que apenas um atendia ao critério de inclusão:

\begin{tabular}{llll}
\hline SMAD 2006 & $\begin{array}{l}\text { Volume } \\
\text { Volumen }\end{array}$ & $\begin{array}{l}\text { Número } \\
\text { Numero } \\
\text { Number }\end{array}$ & $\begin{array}{l}\text { Artigo } \\
\text { Artículo } \\
\text { Article }\end{array}$
\end{tabular}$\quad$ http://www2.eerp.usp.br/resmad/artigos.asp


a) Artigo publicado no Journal of the American Psychoanalytic Association, intitulado “The oral deadlock: treatment of a psychotic child”. Os autores, Anni Bergman, Michael Schwartzman, Phyllis Sloate e Arnold Wilson, descreveram o atendimento de um menino psicótico de 5 anos, assistido em um centro de tratamento universitário para crianças emocionalmente perturbadas, empregando psicoterapia com a mãe e com a criança em sessões de tratamento comum. O plano de tratamento foi tripartite e propunha recriar um laço afetivo mais saudável entre mãe e filho, bem como fomentar um ambiente facilitador a partir do qual a individualização da criança pudesse ocorrer. Empregava três modalidades: 1) psicoterapia psicanaliticamente orientada, na qual mãe e criança eram atendidas por um terapeuta em sessões conjuntas; 2) uma sala de aula terapêutica com programa individualizado para cada criança; 3) sessões individuais com um acompanhante terapêutico.

Terapeuta, professor e acompanhante terapêutico trabalharam em equipe, planejando e coordenando a abordagem total do tratamento sob a supervisão de um analista infantil. O diagnóstico apontou para uma dificuldade instalada na fase de desenvolvimento oral da criança, gerando dificuldades na relação com a mãe em função de um processo de acentuada simbiose e indiferenciação. O conflito vivido pela criança expressava a indecisão entre manter-se dentro da órbita simbiótica ou ousar sair dela e acompanhar seus impulsos de autonomia e individualização. A equipe terapêutica tornou-se a mãe suficientemente boa, que compreendia em que ponto deveria ser dado um “empurrão gentil” para facilitar seu crescimento $^{(11)}$.

Com a prática do AT, como recurso terapêutico, a criança em questão pôde renunciar à simbiose crescente, que teria se tornado sufocante para ela, impedindo seu crescimento. $\mathrm{O}$ at, por passar tanto tempo na casa, assim como nas ruas, na companhia da criança, encontrava-se em posição singular para observar seus comportamentos tanto na presença como na ausência da mãe. As intervenções no lar foram dirigidas às necessidades da criança e à sua capacidade \begin{tabular}{llll}
\hline SMAD 2006 & $\begin{array}{l}\text { Volume } \\
\text { Volumen }\end{array}$ & $\begin{array}{l}\text { Número } \\
\text { Numero } \\
\text { Number }\end{array}$ & $\begin{array}{l}\text { Artigo } \\
\text { Artículo } \\
\text { Article }\end{array}$
\end{tabular} http:/www2.eerp.usp.br/resmad/artigos.asp 
de se mostrar mais autônoma, reforçando-se a capacidade da mãe de permitir esses movimentos evolutivos ${ }^{(11)}$.

Na base de dados PsycINFO foram encontrados três estudos:

a) Artigo original, de autoria de Renata Udler Cromberg, intitulado: “O buraco e a escada manca”, publicado em 1997 na revista Percurso: Revista de Psicanálise, do Instituto Sedes Sapientiae, e também como capítulo do livro Crise e cidade: acompanhamento terapêutico, organizado pela Equipe de Acompanhantes Terapêuticos do Hospital-Dia A Casa, em 1997. A autora relata o atendimento de uma paciente psicótica acompanhada somente em consultório. A partir da necessidade de viagem da analista, foi solicitada a presença de um at. Analista e acompanhante, frente à possibilidade de confusão dos espaços de tratamento, conversavam na busca de delimitação dos espaços, ampliando o recurso terapêutico do AT para além do momento da viagem da analista. A relação entre analista e acompanhante possibilitou o oferecimento de balizas para o atendimento do AT a partir do compartilhamento de parte do material analítico. Mediante o vínculo estabelecido entre at e cliente foi possível a esta sinalizar o momento em que sentia que iria "surtar" novamente ${ }^{(12)}$.

b) Artigo original, publicado no Journal Japanese of Child and Adolescent Psychiatry, em março de 1999, intitulado: "Child therapy in the United States: meeting the needs of children and their parents”, de autoria de Anni Bergman. Sua experiência no City Child Center era psicanaliticamente orientada para o plano de tratamento tripartite: 1) uma sala de aula terapêutica; 2) psicoterapia individual para cada criança e 3) Acompanhamento Terapêutico. O atendimento voltava-se para crianças psicóticas e autistas, economicamente carentes e com perdas traumáticas em seus primeiros anos de vida. O AT era conduzido por estudantes universitários e muitos deles evoluíram depois para programas de $\mathrm{PhD}$ ou se tornaram supervisores. $\mathrm{O}$ atendimento poderia ser feito: 1) nas casas das crianças, mediante

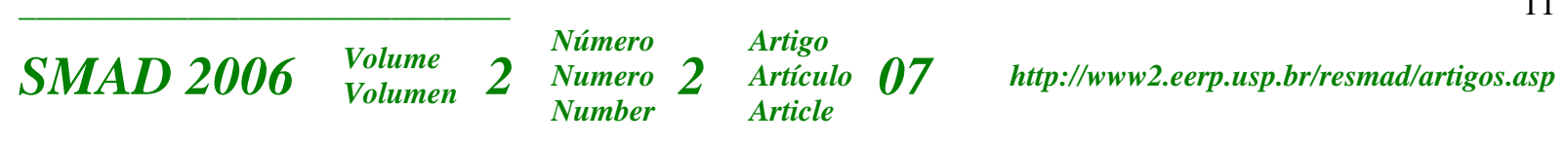


visitas domiciliares, ou levando-as para casa ao final do dia, ou compartilhando refeições com a família. 2) em saídas externas com as crianças, nas quais experimentavam escolher o que queriam comprar, andar de ônibus ou de metrô, ou ir ao parque, objetivando desenvolver capacidades de ego autônomo nas novas experiências ${ }^{(13)}$. O trabalho com at deveria dirigir-se ao isolamento social, buscando anular a tendência de crianças gravemente perturbadas em viver em um mundo muito restrito, ditado pelos seus medos e comportamentos bizarros.

c) Um estudo de caso publicado no Australian and New Zealand Journal of Psychiatry, dos autores Kazuhiko Nakamura, Kazuhiko Iwahashi, Isao Fukunishi e Hiroshi Suwaki, intitulado "Social skills training for a case of Savant syndrome and Asperger's syndrome”. Publicado no ano de 2000, este trabalho relata um caso de síndrome de Asperger em um paciente do sexo masculino de 14 anos, cujo desenvolvimento até os 3 anos de idade foi avaliado como normal. Os critérios de caracterização da síndrome eram observados por meio de testes e abrangiam a habilidade de memorizar mapas. No decorrer do tratamento foi observada a baixa estima do paciente, falta de confiança e pobreza de comunicação com os outros. As intervenções do at no tratamento enriqueciam sua comunicação com o meio social. O reconhecimento de sua habilidade com mapas, encorajando-o a desenhá-los, ampliava a comunicação entre o paciente e o at. Gradualmente foi se configurando sua auto-confiança e o treino de suas habilidades sociais melhorou sua capacidade de comunicação, favorecendo seu desempenho no exame de admissão no ginásio, que o levou a ser aceito em três escolas, além de incluir-se em atividades atléticas no clube e fazer amizades ${ }^{(14)}$.

\section{CONSIDERAÇÕES FINAIS}

$\mathrm{Na}$ análise dos estudos selecionados observamos que o AT corresponde a uma prática clínica em construção, que admite a utilização de diversos referenciais teóricos e inúmeras possibilidades de experiências clínicas em diferentes contextos de intervenção. Sua

\begin{tabular}{llll}
\hline SMAD 2006 & $\begin{array}{l}\text { Volume } \\
\text { Volumen }\end{array}$ & $\begin{array}{l}\text { Número } \\
\text { Numero } \\
\text { Number }\end{array}$ & $\begin{array}{l}\text { Artigo } \\
\text { Artículo } \\
\text { Article }\end{array}$
\end{tabular} http://www2.eerp.usp.br/resmad/artigos.asp


aplicabilidade se estende desde pacientes psicóticos, adultos e crianças, portadores de deficiências, assim como suicidas e drogaditos. A influência das idéias do movimento da antipsiquiatria foi marcante nas origens do AT, na medida em que revolucionaram o conceito de doença mental e favoreceram a quebra dos dogmas de exclusão, isolamento e dor.

A década de 80 marcou o início da estruturação da prática clínica do AT, mediante o estabelecimento de uma sistematização necessária à formação teórica e prática do at, enfatizando-se o trabalho terapêutico pessoal do profissional e a supervisão dos atendimentos. Atualmente, a prática do AT tem se legitimado na abertura de novas possibilidades de ação profissional na área da saúde, caracterizada pela ação interdisciplinar e pelo modelo de atenção psicossocial.

Podemos perceber nos estudos revisados a preocupação com a formalização e delineamento do trabalho desenvolvido na clínica do AT, na qual o terapeuta at assume uma função de aliado do cliente, uma espécie de ego auxiliar, um aporte que permite constituir um nível econômico de energia que ajuda a drenar o sofrimento do acompanhado. Na medida em que o cliente se sente compreendido, possibilita-se o reconhecimento de sua identidade e a reconstrução de sua auto-estima, que o leva a recuperar a esperança e a confiança em seus próprios recursos adaptativos. Constrói-se, assim, uma intervenção que enfatiza a importância da elaboração de um novo projeto de vida para os portadores de dificuldades psicossociais.

\section{REFERÊNCIAS BIBLIOGRÁFICAS}

1- Mauer S, Resnizky S. Acompanhamento terapêutico e pacientes psicóticos: manual introdutório a uma estratégia clínica. Campinas (SP): Papirus; 1987.

2- Equipe de Acompanhantes Terapêuticos do Hospital-Dia A Casa (Organizadores). A rua como espaço clínico: acompanhamento terapêutico. São Paulo (SP): Escuta; 1991.

3- Equipe de Acompanhantes Terapêuticos de A Casa (Organizadores). Crise e cidade: acompanhamento terapêutico. São Paulo (SP): EDUC; 1997. 
4 - Reis R. Neto - O acompanhante terapêutico: trajetória histórica de uma prática em saúde mental no Rio de Janeiro. Rio de Janeiro (RJ): Departamento de Psicologia, Pontifícia Universidade Católica do Rio de Janeiro; 1995.

5- Sereno D. Acompanhamento terapêutico de pacientes psicóticos: uma clínica na cidade. [dissertação]. São Paulo (SP): Instituto de Psicologia da Universidade de São Paulo; 1996.

6- Barreto KD. Andanças com Dom Quixote e Sancho Pança pelos caminhos da transicionalidade: relatos de um acompanhante terapêutico. [dissertação]. São Paulo (SP): Pontíficia Universidade Católica de São Paulo/PUC; 1997.

7- Lerner BD. Nuevo modo de investigar en psiquiatria: el acompañamiento terapéutico. Acta Psiquiatr. Psicol. Am. Lat. 1984 julho; 30(1): 21-8.

8- Lerner BD. Como acompañar a un suicida. Acta Psiquiatr. Psicol. Am. Lat 1986 junho; 32(2): 137-47.

9- Antonucci R. Terapias ressocializantes: o acompanhante terapêutico. In: Assumpçäo Jr. FB (org.). Psiquiatria da infância e da adolescência. Santos (SP): Summus; 1994. p. 549-55.

10- Ayub P. Do amigo qualificado ao acompanhante terapêutico. Infanto Rev Neuropsiquiatria da Infância e Adolescência 1996 agosto; 4(2):37-40.

11- Bergman A, Schwartzman M, Sloat P, Wilson A. The oral deadlock: treatment of a psychotic child. J. Am Psychoanalytic Assoc. 1983. 31(2): 443/65.

12- Cromberg RU. O buraco e a escada manca. Percurso: Revista de Psicanálise 1997; 9(18)[1]: 59-64.

13- Bergman A. Child therapy in the United States: meeting the needs of children and their parents. Japanese J. Child and Adolescence Psychiatry 1999 march-april; 40(2): 120133.

14- Nakamura K, Iwahashi K, Fukunishi I, Suwaki H. Social skills training for a case of Savant syndrome and Asperger's syndrome. Austr. N. Z. J. Psychiatry, 2000 August; 34(4): 697. 